\author{
Revista Venezolana de Gerencia (RVG) \\ Año 17. N 57, 2012, 9 - 38 \\ Universidad del Zulia (LUZ) • ISSN 1315-9984
}

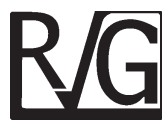

\title{
Factores del entorno y creación de empresas: un análisis institucional*
}

\author{
Alvarez, Claudia** \\ Urbano, David***
}

\section{Resumen}

Dado el relevante papel que juega la actividad emprendedora en el desarrollo económico y social de países y regiones, es necesario seguir avanzando en sus determinantes. Así pues el objetivo de este trabajo es analizar la influencia que tienen los factores del entorno en la creación de empresas, utilizando como marco teórico de referencia la teoría económica institucional. A partir de un modelo de datos de panel y con información del proyecto Global Entrepreneurship Monitor (GEM), se demuestra estadísticamente que instituciones como estabilidad política y modelos de referencia están relacionadas con la creación de empresas. Los resultados de este trabajo indican que en los países de mediano y bajo ingreso, los factores informales (estabilidad política y modelos de referencia) tienen mayor impacto en la creación de empresas; mientras que en el caso de los países de ingreso alto son más determinantes los factores formales (procedimientos para crear empresa y formación en gestión y administración). Dichas evidencias pueden ser muy útiles para el diseño de políticas gubernamentales relativas al fomento de la actividad emprendedora.

Palabras clave: Creación de empresas, actividad emprendedora, factores del entorno, instituciones, teoría económica institucional, Global Entrepreneurship Monitor.

Recibido: 26-11-10. Aceptado: 09-10-11

* Los autores agradecen los comentarios a las versiones previas del trabajo por parte de Josep Lluís Raymond y Stefan Van Hemmen (Universidad Autónoma de Barcelona) y Joan Manel Batista (ESADE), así como las aportaciones de los evaluadores anónimos del artículo. Además, destacan el apoyo financiero de los proyectos ECO2010-16760 (Ministerio de Ciencia e Innovación de España) y 2005SGR00858 (Departamento de universidades, investigación y sociedad de la información de Catalunya).

** Doctora en Creación y Gestión de Empresas de la Universidad Autónoma de Barcelona, España. Profesora e investigadora de la Universidad de Medellín, Colombia. e-mail: claudiapatricia.alvarez@uab.es.

*** Doctor en Administración y Dirección de Empresas (European Doctoral Programme in Entrepreneurship and Small Business Management - UAB-Växjö University). Profesor e investigador de la Universidad Autónoma de Barcelona, España. e-mail: david.urbano@uab.es. Autor para la correspondencia. 


\title{
Environmental Factors and New Firm Creation: An Institutional Approach
}

\begin{abstract}
Given the relevant role that entrepreneurship plays in the economic and social development of countries and regions, further progress regarding its determinants is needed. The aim of this study is to analyze the influence that environmental factors have on creating new firms, using institutional economics as a theoretical framework. A panel data model with information from the Global Entrepreneurship Monitor (GEM) is used to prove statistically that institutions like political stability and role models are related to creating enterprises. The main findings indicate that in medium and low income countries, informal factors (political stability and role models) have a greater impact on entrepreneurship, while in high income countries, the formal factors (procedures for starting businesses and training in management and administration) are more determinant. These findings also have several practical implications for policy makers in terms of designing governmental initiatives to promote entrepreneurship.
\end{abstract}

Key words: New firm creation, entrepreneurial activity, environmental factors, institutions, institutional economics, Global Entrepreneurship Monitor.

\section{Introducción}

Si bien tradicionalmente el crecimiento económico estuvo asociado con el dinamismo de las grandes empresas, a partir de la década de los 80's viene dándose una importancia creciente a la contribución que las pequeñas y medianas empresas hacen a la economía mundial, destacando especialmente el papel de las nuevas empresas como motor de desarrollo económico y social (Wennekers et al., 1999 y 2005; Audretsch y Keilbach, 2004; Van Stel et al., 2005; Acs et al., 2007 y 2008). A partir de dicho reconocimiento, las diferentes instancias gubernamentales han mostrado particular interés en el diseño de políticas y estrategias de fomento a la actividad emprendedora, a la vez que la academia se ha interesado por el fenómeno emprendedor, especialmente por los factores que condicionan la creación de empresas como objeto de estudio.
Varios son los autores que han venido trabajando sobre esta temática en las últimas décadas (Gartner, 1985; Brockhaus, 1987; Johannisson, 1988; Bygrave y Hofer, 1991; Gnyawali y Fogel, 1994; Shane y Venkataraman, 2000; Verheul et al., 2001; Busenitz et al., 2003; Davidsson, 2003; Steyaert y Hjorth, 2006; entre otros). Desde una perspectiva muy general, podría decirse que las investigaciones en el campo del emprendimiento se han situado en tres grandes enfoques. Por una parte, el enfoque económico, donde se imponen los aspectos relativos a la racionalidad económica y que, a grandes rasgos, plantea que el emprendimiento obedece a cuestiones puramente económicas (Audretsch y Thurik, 2001; Audretsch y Keilbach, 2004; Parker, 2004; Wennekers et al., 2005, entre otros). Por otra, el enfoque psicológico postula que son los factores individuales o rasgos psicológicos de las personas (McClelland, 1961; Collins et al., 1964; Carsrud y Johnson, 1989; entre otros) los que determinan 
la actividad emprendedora. Por último, el enfoque sociológico o institucional defiende que es el entorno socio-cultural (Shapero y Sokol, 1982; Aldrich y Zimmer, 1986; Berger, 1991; Busenitz et al., 2000; Steyaert y Katz, 2004; Manolova et al., 2008; Gómez-Haro y Salmeron-Gómez, 2011) el que condiciona la decisión de crear una empresa.

En el presente trabajo se toma como marco conceptual esta última aproximación. Concretamente se consideran los planteamientos de la teoría económica institucional (North, 1990 y 2005) aplicada al análisis de la creación de empresas. En este contexto, son los factores del entorno o institucionales los que condicionan el emprendimiento, distinguiendo entre factores formales (organismos y medidas de apoyo a la creación de empresas, trámites y costes para iniciar una actividad empresarial, entre otros) y factores informales (modelos de referencia de empresarios, espíritu emprendedor, actitudes hacia el emprendimiento, entre otros.). Además, con la finalidad de aproximarnos a la operacionalización de dichos factores se consideran las dimensiones del entorno de Gnyawali y Fogel (1994) (políticas y procedimientos gubernamentales, condiciones sociales y económicas, conocimientos y habilidades emprendedoras, asistencia financiera para emprender y asistencia no financiera).

En este sentido, el objetivo principal de este trabajo es analizar la influencia que tienen los factores del entorno en la creación de empresas. Dichos factores se operacionalizaron a través de las instituciones informales (estabilidad política, control de la corrupción, modelos de refe- rencia) y las formales (procedimientos en creación de empresas, formación en gestión y administración, acceso al crédito bancario). Así pues, a partir de un modelo de datos de panel correspondiente al periodo 2004-2009 y con información del proyecto Global Entrepreneurship Monitor (GEM), se demuestra estadísticamente el impacto que algunas instituciones tienen en la creación de empresas.

En cuanto a las implicaciones de la investigación, los resultados obtenidos permiten por un lado, avanzar en la consolidación de la teoría económica institucional como marco conceptual para el análisis de la creación de empresas, a la vez de reafirmar y validar empíricamente la importancia de los factores del entorno en el proceso de creación de empresas. Cabe decir, que a pesar de que cada día son más los investigadores que se decantan por el enfoque institucional, no existen aún demasiados trabajos empíricos a la luz de esta teoría, seguramente por las dificultades relacionadas con la medición de las variables relativas al marco institucional. Además, su aplicación no es clara o directamente relevante para la investigación en creación de empresas (Bruton et al., 2010).

Por otro lado, las evidencias encontradas pueden ser muy útiles para el diseño de políticas gubernamentales relativas al fomento de la creación de empresas acordes a las características específicas de los distintos países analizados.

\section{Factores del entorno y creación de empresas}

Las instituciones han sido creadas como mecanismo para reducir la incerti- 
dumbre en la vida diaria y dar forma a la interacción humana. Se definen como reglas de juego que regulan las relaciones políticas, sociales y económicas, proporcionando estructura y orden a dichos intercambios (North, 1990). Así mismo, las instituciones pueden ser formales, referidas a reglamentaciones políticas, legales, económicas y contratos diseñados para delimitar (en términos legales) el comportamiento de los individuos y facilitar el intercambio político y económico; o informales, que incluyen las convenciones sociales, valores, ideas, actitudes, percepciones subjetivas y símbolos no escritos compartidos por una sociedad, que coordinan la interacción social y afectan la aplicación, interpretación y cumplimiento de las normas formales, es decir, lo que es permisible o no en las relaciones humanas (North, 1990 y 2005).

De igual forma, las instituciones formales e informales se complementan para definir el conjunto de elecciones realizadas y resultados obtenidos en una sociedad. Por lo tanto analizar únicamente el entorno formal daría una noción insuficiente e inadecuada de la relación existente entre las instituciones y el desempeño observado. En este sentido, hay evidencia de que las mismas normas formales impuestas en sociedades distintas producen resultados diferentes (North, 1990 y 2005).

Se puede decir, entonces, que la conducta humana está condicionada por el entorno institucional. Así pues, las instituciones formales e informales pueden li- mitar o favorecer las decisiones de los individuos y específicamente la decisión de crear o no una empresa.

A su vez, siguiendo con el enfoque institucional, varios autores (Welter, 2005 y 2011; Urbano, 2006; Aidis et al., 2008; Veciana y Urbano, 2008; Stephen et al., 2009; Thornton et al., 2011; Alvarez y Urbano, 2011b, Welter y Smallbone, 2011; entre otros), proponen en sus trabajos la aplicación de los planteamiento de North (1990 y 2005) para el análisis de la creación de empresas. En este sentido, en la Figura 1 se observa en la parte izquierda el esquema de North (1990 y 2005), mientras que en la parte derecha puede verse como el empresario, en su papel de responsable y catalizador en el proceso de creación de empresas, está condicionado por los factores del entorno, tanto formales como informales, y es el encargado de implementar las normativas y reglamentaciones relacionadas con la actividad emprendedora, así como las normas informales fruto de su aprendizaje y del proceso de socialización, contando además con la incidencia de otro tipo de organizaciones, políticas, económicas, sociales y educativas.

Por otro lado, y ya en el ámbito específico de la literatura sobre creación de empresas y los factores del entorno, Gnyawali y Fogel (1994) consideran cinco dimensiones del entorno que condicionan la actividad emprendedora, a saber: a) políticas y procedimientos gubernamentales, b) condiciones sociales y económicas ${ }^{1}$, 


\section{Figura 1 \\ Aplicación de los planteamientos de North a la creación de empresas}

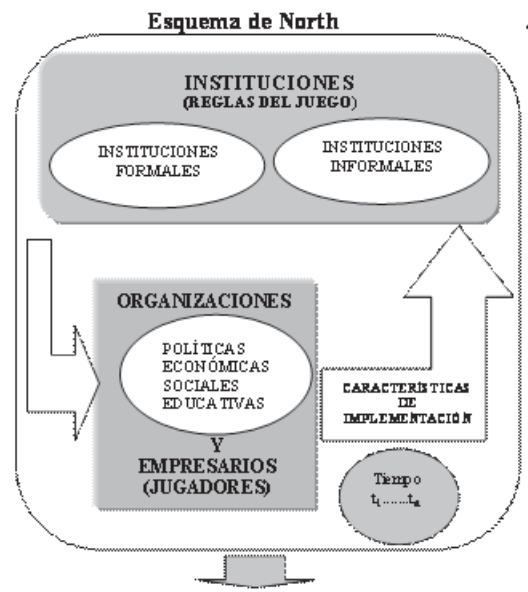

CAMBIO INSTITUCIONAL $\Longrightarrow$ DESEMPEÑO ECONOMICO

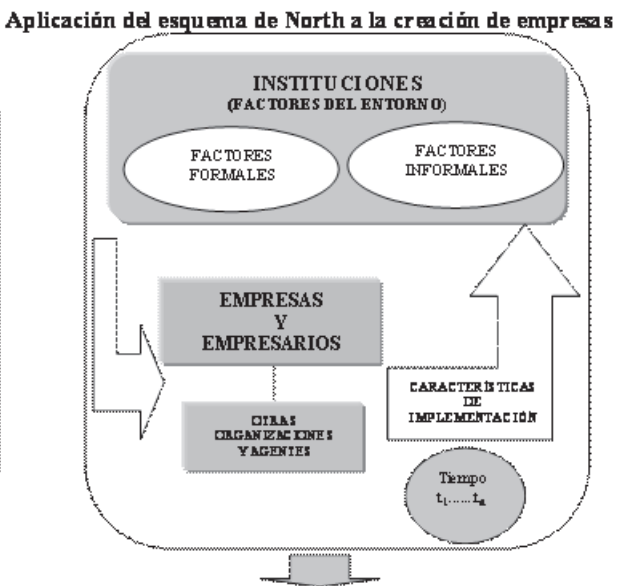

CREACIONDE EMPRESAS $\rightleftarrows$ CRECTMIENTO Y DESARROLLO ECONÓMICO

Fuente: Urbano et al., 2007.

c) conocimientos y habilidades emprendedoras, d) asistencia financiera para emprender, e) asistencia no financiera.

Por políticas y procedimientos gubernamentales se entienden todas aquellas acciones de los gobiernos que pueden influir en los mecanismos de mercado, haciendo que funcionen eficientemente, mediante la eliminación de las imperfecciones de dicho mercado y a través de regulaciones administrativas más o menos rígidas, por ejemplo, por medio de subvenciones para fomentar la creación de empresas, regulación laboral, impuestos y trámites para abrir un negocio, políti-

cas de fomento del emprendimiento en colectivos específicos (mujeres, jóvenes, inmigrantes), entre otros. En cuanto a las condiciones sociales, principalmente se consideran las actitudes favorables hacia la actividad emprendedora, la presencia de emprendedores con experiencia, los modelos de referencia exitosos, los antecedentes de empresarios en la familia, la valoración de la figura del empresario en la sociedad, entre otros. A su vez, los conocimientos y habilidades emprendedoras se consideran como las competencias técnicas necesarias para iniciar una nueva empresa, adquiridas a través de

nómico de la región o país y la diversidad de actividades económicas) con la finalidad de adaptarlo al marco conceptual utilizado. 
programas de entrenamiento y formación $^{2}$, ya sean específicos para la elaboración de planes de negocio, o de carácter más general del ámbito de la gestión empresarial. Así mismo, los emprendedores requieren tanto asistencia financiera como no financiera. Es decir, por un lado, financiación para lanzar su negocio, diversificar el riesgo de emprender, crecer y expandirse. Por el otro, servicios no económicos, por ejemplo, realizar estudios de mercado, preparar el plan de empresa, acceder a contactos y redes con otros emprendedores, entre otros.

Específicamente, siguiendo las dimensiones propuestas por Gnyawali y Fogel (1994) y adaptándolas a los planteamientos de North (1990 y 2005), fácilmente se puede ver que las políticas y procedimientos gubernamentales, los conocimientos y habilidades emprendedoras, $y$ la asistencia financiera y no financiera se refieren a los factores formales, mientras que las condiciones sociales corresponden a los factores informales. En el Cuadro 1 se presentan los estudios más relevantes sobre la relación entre los factores del entorno y la actividad emprendedora utilizados en este trabajo (para más detalle ver Alvarez y Urbano, 2011a).

En referencia a los factores informales, una de las formas utilizadas por el Banco Mundial para medir las instituciones ha sido por medio del concepto de gobernabilidad, entendida como las tradiciones e instituciones a través de las cuales se ejerce la autoridad en un país, in- cluyendo el mecanismo de elección del gobierno, su control y sustitución; la capacidad del gobierno para formular y aplicar buenas políticas y el respeto de los ciudadanos y el estado mismo por las instituciones que regulan sus interacciones económicas y sociales (Kaufmann et al., 2008). Asimismo, cada vez es mayor el número de investigaciones que presentan una relación positiva entre la gobernabilidad e indicadores como el crecimiento del PIB per cápita (Kaufmann y Kraay, 2003), el Índice de Desarrollo Humano de las Naciones Unidas (RoseAckerman, 2004), la inequidad del ingreso (Li, Xu y Zou, 2000; Carmignani, 2005) y la actividad emprendedora (Johnson et al., 1999 y 2000; McMillan y Woodruff, 1999 y 2002; Djankov et al, 2005, 2006).

Aunque la forma precisa de la relación varía entre dichos estudios (algunos sugieren que es positiva y directa, mientras que otros apuntan que es positiva, pero curvilínea), un reciente metaanálisis de 89 estudios confirmó que la gobernabilidad (de la cual el control de la corrupción y la estabilidad política son componentes muy importantes), modera los principales indicadores económicos $\mathrm{y}$, por lo tanto, desempeña un papel primordial en el desarrollo económico de los países y regiones (Doucouliagos y Ulubasoglu, 2008; Kaufmann et al., 2008).

Así pues, bajos índices de gobernabilidad implican normalmente conductas que afectan la confianza depositada

2 En este artículo se considera que los conocimientos y habilidades emprendedoras son un factor formal cuando son adquiridos a través de formación reglada. 


\section{Cuadro 1 \\ Selección de trabajos más relevantes que relacionan los factores del entorno y la actividad emprendedora}

\begin{tabular}{|c|c|c|c|}
\hline $\begin{array}{l}\text { Instituciones } \\
\text { según North }\end{array}$ & $\begin{array}{l}\text { Dimensiones según } \\
\text { Gnyawali y Fogel }\end{array}$ & $\begin{array}{l}\text { Variables } \\
\text { del trabajo* }\end{array}$ & $\begin{array}{l}\text { Trabajos empíricos } \\
\text { (por autores) }\end{array}$ \\
\hline $\begin{array}{l}\text { Factores } \\
\text { del entorno } \\
\text { Instituciones } \\
\text { Informales }\end{array}$ & $\begin{array}{c}\text { Condiciones } \\
\text { sociales }\end{array}$ & $\begin{array}{l}\text { EP } \\
\text { CC } \\
\text { MR }\end{array}$ & $\begin{array}{l}\text { Aidis et al., 2008; Alvarez et al., 2011; } \\
\text { Anokhin y Schulze, 2009; Arenius y De } \\
\text { Clercq, 2005; Arenius y Kovalainen, 2006; } \\
\text { Arenius y Minniti, 2005; Baughn et al, } \\
\text { 2006; Begley et. al., 2005; Begley y Tan, } \\
\text { 2001; Brush, 1992; Davidson, 1995; De } \\
\text { Clercq y Arenius, 2006; Koellinger y Min- } \\
\text { niti, 2006; Koellinger, 2008; Krueger et al, } \\
\text { 2000; Kwon y Arenius, 2010; Langowitz y } \\
\text { Minniti, 2007; Levie, 2007; Minniti y Nar- } \\
\text { done, 2007; Reynolds y White, 1997; } \\
\text { Shane et al., 1991; Shapero y Sokol, } \\
\text { 1982; Thompson et al 2009; Tominc y Re- } \\
\text { bernik, 2007; Vaillant y Lafuente, 2007; } \\
\text { Verheul et al, 2006; Wagner, 2007. }\end{array}$ \\
\hline
\end{tabular}

Factores

del entorno

Instituciones

Formales

$$
\text { Políticas y }
$$

procedimientos

de gobierno

Asistencia

financiera y no

financiera

Conocimientos

y habilidades

emprendedoras
PC

Audrestch, 2004; Bruno y Tyebjee, 1982;

Gnyawali y Fogel, 1994; Ho y Wong, 2007; Lundstrom and Stevenson, 2005; Marshall et al., 1993; McMullen et al., 2008; Spilling, 1996; Stephen et al., 2005 y 2009; Storey, 1994; Van Stel et al, 2007.

AC Ang, 1991; Bates, 1997; George y Prabhu, 2003; Kortum y Lerner, 2000; Mason y Harrinson, 1994; Maula et al, 2005; Naude et al, 2008; Ritsilä y Tervo, 2002; Roper y Scott, 2009; Sarder et al, 1997; Szerb et al, 2007; Trulsson, 2002; Wetzel, 1987.

FG Audrestch, 2004; Bennet y Robson, 1999; Dana, 1996; Dolinsky et al, 1993; Gartner y Vesper, 1994; Gorman et. al, 1997; McQuaid, 2002; Olsson, 2000; Ronstadt, 1985; Shane y Kolvereid, 1995.

*(EP) Estabilidad Política, (CC) Control de la Corrupción, (MR) Modelos de Referencia de Empresarios, (PC) Procedimientos Creación de Empresas, (AC) Acceso al Crédito Bancario, (FG) Formación en Gestión y Administración de Empresas.

Fuente: Elaboración propia. 
en los funcionarios públicos y, por lo tanto, socavan las bases de la confianza interpersonal, dificultando la creación de empresas pues en dicho proceso es esencial la capacidad de confiar en otras personas. Inestabilidad política y altos niveles de corrupción incrementan el riesgo del empresario ya que otros agentes implicados en su cadena de valor podrían tener comportamientos oportunistas y aprovecharse de sus beneficios o rentas; situaciones que son factibles debido a los niveles relativamente elevados de asimetrías de información que caracterizan a cualquier actividad económica (Anokhin y Schulze, 2009).

Además, la inestabilidad política aumenta la incertidumbre en la economía y disuade a los emprendedores interesados en aprovechar oportunidades de negocio (Aysan et al., 2007). En contraste, entornos caracterizados por altos niveles de confianza pueden facilitar los intercambios comerciales ya que las partes que intervienen en las transacciones tienden a compartir valores similares, los canales de comunicación son más eficientes y además existen mecanismos que posibilitan la solución de controversias y reducen sus costes (Rose-Ackerman, 2001). Estudios comparativos a nivel país muestran relaciones positivas entre la estabilidad política y la inversión (Wohlgemuth, 2000).

Asimismo, indicadores multidimensionales de credibilidad en las leyes, reglamentos y políticas, que han sido asociados a la estabilidad política, también muestran relaciones positivas con la inversión privada, la actividad emprendedora y el crecimiento económico. Johnson et al. (2002) analizaron la creación de empresas en países post-comunistas y encontraron que los pagos extralegales (sobornos) y la ineficiencia de los tribunales inhiben la iniciativa emprendedora más que la falta de financiación. Se deduce entonces que la corrupción y la inestabilidad política, al igual que otras deficiencias en la infraestructura de un país, aumentan los costos de transacción y de agencia a la vez que limitan los ingresos. Por el contrario, el control de la corrupción y la estabilidad política aumentarian la probabilidad de que los futuros empresarios capturen una mayor porción de los ingresos que generan, aumentarían la fiabilidad de los flujos de efectivo y, por lo tanto, motivarían a niveles más altos de creación de empresas. En consecuencia, se plantean las siguientes hipótesis:

$\mathrm{H}_{1}$ : Existe una relación positiva entre la estabilidad política y la creación de empresas

$\mathrm{H}_{2}$ : Existe una relación positiva entre el control de la corrupción y la creación de empresas

Otro factor informal considerado en este trabajo son los modelos de referencia (Shapero y Sokol, 1982; Krueger, 1993), a los que se atribuye una gran importancia en la decisión de crear una empresa, bajo el argumento que las personas que se han socializado entre empresarios, perciben menor nivel de incertidumbre, tienen más confianza en su función de emprendedores (Bandura, 1978), cuentan con mayores probabilidades de crear una empresa y además suelen ver como más viable la continuidad de la empresa una vez creada (Davidsson y Honig, 2003). La literatura ha presentado evidencias sobre la relación positiva entre la presencia de modelos de referencia familiares y los nuevos emprendedores. 
Collins et al. (1964) fueron los primeros autores en verificar empíricamente la influencia del contexto familiar sobre los emprendedores y sus empresas. Otros trabajos más recientes son Brockhaus y Horwitz (1986), Cooper (1986), Matthews y Moser (1995), y Scott y Twomey (1988). En líneas generales podría decirse entonces que la presencia de emprendedores con experiencia y los modelos de referencia exitosos transmiten mensajes positivos a los potenciales emprendedores (Gnyawali y Fogel, 1994). Por lo tanto:

$\mathrm{H}_{3}$ : Existe una relación positiva entre los modelos de referencia de empresarios y la creación de empresas

En cuanto a los factores formales, Gnyawali y Fogel (1994) afirman que la regulación gubernamental en el ámbito económico puede ser percibida negativamente, especialmente por aquellos interesados en crear nuevas empresas. De igual forma, la evidencia empírica sugiere que los emprendedores pueden declinar sus intenciones de crear una empresa si tienen que seguir muchas reglas y procedimientos, obtener gran número de licencias y cumplir con demasiados trámites (Dana 1990; Young y Welch, 1993; Begley et al., 2005). Bajo estos supuestos el proyecto Doing Business del Banco Mundial proporciona anualmente mediciones objetivas de las regulaciones para hacer negocios y su aplicación en 183 países. El proyecto alienta cada año a los países a disminuir la regulación, pues procedimientos sencillos de apertura podrían estimular la creación de nuevas empresas. Según el informe Doing Business 2009, estudios realizados en México, India, Brasil y Rusia concluyen que un régimen simplificado de acceso se asocia a un nú- mero mayor de empresas registradas y facilitan que los trabajadores y el capital se muevan entre los distintos sectores cuando las economías experimentan momentos difíciles. De hecho, simplificar las formalidades de registro fue la reforma más popular durante los años 2007 y 2008, implementada en 49 países. Así pues, se formula la siguiente hipótesis:

$\mathrm{H}_{4}$ : Existe una relación negativa entre el número de procedimientos para crear una empresa y la creación de empresas.

Por otro lado, la literatura considera de gran importancia los aspectos relativos a la financiación de las nuevas empresas. En un estudio realizado a partir de una muestra de personas que contaban con ideas de negocio pero que finalmente no crearon una empresa, Van Auken (1999) encontró que las restricciones financieras fueron el obstáculo principal. Los recursos necesarios para iniciar una actividad empresarial están, por lo general, fuera del alcance de los empresarios individuales (Bhave, 1994). En este sentido, la falta de financiación podría ser una razón para que los potenciales emprendedores abandonen el proyecto de crear una empresa (Holtz-Eakin et al., 1994; Blanchflower y Oswald, 1998). A su vez, diversos estudios han comprobado que las políticas orientadas a aumentar el acceso al crédito bancario mediante la disminución de requisitos de capital, creación de compañías de inversión, créditos con bajas tasas de interés y sistemas de garantía de crédito, contribuyen de forma relevante al fomento de nuevas empresas (Gnyawali y Fogel, 1994; Van Gelderen et al., 2006). En consecuencia: 
$\mathrm{H}_{5}$ : Existe una relación positiva entre el acceso al crédito bancario y la creación de empresas

El último factor formal tiene que ver con los conocimientos y habilidades emprendedoras. En términos generales, la literatura sostiene que habitualmente los comportamientos de las personas estarian dirigidos por sus competencias. Específicamente, resultados de investigaciones recientes muestran que elevados niveles de educación tienen un efecto positivo sobre la probabilidad de crear una empresa (Delmar y Davidsson, 2000; Davidsson y Honig, 2003; Arenius y De Clercq, 2005; Arenius y Minniti, 2005; De Clerq y Arenius, 2006; Levie y Autio, 2008).

Siguiendo esta misma línea, otros estudios han encontrado que un bajo nivel de habilidades en gestión y administración puede inhibir a los emprendedores que van a crear su empresa (Davidsson, 1991; Gnyawali y Fogel, 1994).
Así pues, los individuos podrían estar más inclinados a tomar la decisión de crear una empresa si creen que tienen los conocimientos necesarios para ello (Arenius y Minniti, 2005; Boyd y Vozikis, 1994; Chen et al., 1998; Davidsson y Honig, 2003; Scott y Twomey, 1988). De esta manera se plantea la siguiente hipótesis:

$\mathrm{H}_{6}$ : Existe una relación positiva entre el nivel de formación y gestión en administración de empresas y la creación de empresas

A continuación, en la Figura 2 se presenta el modelo teórico de esta investigación con las respectivas hipótesis.

\section{Factores del entorno para la creación de empresas: la evidencia empírica}

Tal como ya se comentó anteriormente, este trabajo analiza la relación en-

\section{Figura 2}

\section{Los factores del entorno para la creación de empresas $y$ el enfoque institucional}

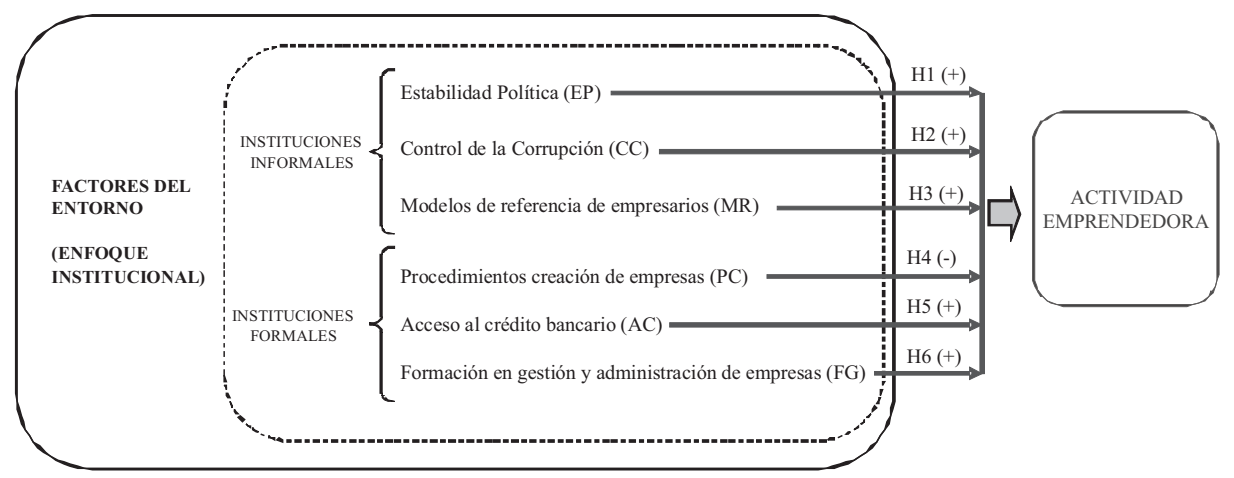

Fuente: Elaboración propia. 
tre los factores del entorno y la creación de empresas, por medio de un panel de datos para el periodo 2004-2009.

La fuente de información utilizada para medir la variable dependiente es la tasa de actividad emprendedora (TEA), uno de los indicadores más conocidos del proyecto Global Entrepreneurship Monitor (GEM), que considera como emprendedores a aquellos adultos (entre 18 y 64 años) que se hallan inmersos en el proceso de puesta en marcha de una empresa o que han iniciado una actividad empresarial en los últimos 42 meses. El proyecto GEM es actualmente el estudio más relevante sobre la actividad emprendedora en el mundo, surgido como una investigación conjunta entre las universidades London Business School (Reino Unido) y la Babson College (EEUU), con el fin de estudiar la relación entre la creación de empresas y el crecimiento económico. En la primera versión realizada en 1999 contó con la participación de 10 países, mientras que la última investigación llevada a cabo en el año 2009 fue realizada en 54 países. La muestra final de este estudio está formada por un panel desbalanceado de 243 observaciones y 70 países $^{3}$ (ver Cuadro 2).

En cuanto a las variables independientes, por el lado de las instituciones informales, la estabilidad política (EP) y el control de la corrupción (CC) proceden del proyecto de investigación Worldwide Governance Indicators (WGI). Este proyecto refleja el conjunto de tradiciones e instituciones por las que se ejerce la autoridad en un país, incluyendo el proceso por el cual los gobiernos son elegidos, supervisados y reemplazados, la capacidad del gobierno para formular y aplicar políticas eficaces, y el respeto de los ciudadanos y el estado a las instituciones que rigen sus interacciones económicas y sociales (Kaufmann et al., 2008). Los indicadores de gobernabilidad cubren 213 países y están basados en 33 fuentes que incluyen una recopilación de más de 120000 respuestas proporcionadas por ciudadanos, expertos y empresas en todo el mundo. Cada indicador tiene un rango de unidades que va de -2.5 a 2.5 , donde los valores más elevados corresponden a mejores resultados de las instituciones. Específicamente, la estabilidad política se refiere a la ausencia de violencia, medida como la probabilidad de que el estado esté sujeto a amenazas violentas o cambios. A su vez, el control de la corrupción se refiere al abuso del poder público en beneficio del privado, incluyendo la corrupción a pequeña y gran escala y el control del estado por las élites. Asimismo, la variable modelos de referencia de empresarios (MR) se obtiene también de la base de datos del proyecto GEM y se refiere al porcentaje de indivi-

3 Aunque el panel de datos cubre el periodo 2004-2009, el número de observaciones está condicionado por la regularidad en la que los países participan anualmente en el GEM. Así pues, hay países como España que participan cada año, otros países como Hong Kong que han participado tres años, o Austria que tan sólo ha participado dos veces en dicho periodo. 


\section{Cuadro 2}

\section{Lista de países clasificados por nivel de ingresos}

\begin{tabular}{|c|c|c|c|}
\hline ngreso alto: OCDE & Ingreso alto: No OCDE & Ingreso medio-alto & Ingreso medio-bajo y \\
\hline Alemania & Arabia Saudita & Argelia & Bolivia \\
\hline Australia & Croacia & Argentina & Cisjordania y Franja de Gaza \\
\hline Austria & Emiratos Árabes Unidos & Bosnia y Herzegovina & China \\
\hline Bélgica & Eslovenia & Brasil & Ecuador \\
\hline Canadá & Hong Kong & Colombia & Egipto \\
\hline Corea & Israel & Chile & Filipinas \\
\hline España & Puerto Rico & Jamaica & Guatemala \\
\hline Finlandia & Singapur & Kazajstán & India \\
\hline Grecia & & Letonia & Indonesia \\
\hline Hungría & & Líbano & Irán \\
\hline Islandia & & Macedonia & Jordán \\
\hline Irlanda & & Malasia & Siria \\
\hline Italia & & México & Tailandia \\
\hline Japón & & Panamá & Tonga \\
\hline Nueva Zelanda & & Perú & Túnez \\
\hline Noruega & & Polonia & Uganda \\
\hline Países Bajos & & República Dominicana & Yemen \\
\hline Portugal & & Rumania & \\
\hline Reino Unido & & Rusia & \\
\hline República Checa & & Serbia & \\
\hline Suecia & & Sur África & \\
\hline Suiza & & Turquía & \\
\hline & & Uruguay & \\
\hline & & Venezuela & \\
\hline
\end{tabular}

Fuente: World Bank (2011).

duos que conoce personalmente a alguien que ha iniciado un negocio en los últimos dos años (Reynolds et al., 2005).

A su vez, en el caso de las instituciones formales, los procedimientos para la creación de empresas (PC) se tomaron del proyecto Doing Business del Banco Mundial, que proporciona una medición objetiva de las regulaciones para hacer negocios y su respectiva aplicación en 183 países. Específicamente la variable $\mathrm{PC}$ se refiere al logaritmo natural del pro- ducto entre el número de procedimientos que se requieren oficialmente para que un empresario pueda abrir y operar una empresa industrial o comercial y la duración de dichos procedimientos. Asimismo, la variable de acceso al crédito (AC) se obtuvo del indicador de crédito interno provisto por el sector bancario del Banco Mundial. Dicho indicador incluye todo el crédito a los distintos sectores. Los datos sobre la formación en gestión y administración de empresas (FG) se obtuvieron 
de la base de datos de la UNESCO, a partir del producto resultante entre el porcentaje de universitarios con respecto a la población y el porcentaje de universitarios en áreas de ciencias sociales, gestión y derecho.

Finalmente, teniendo en cuenta que el nivel de desarrollo económico de los países y específicamente la renta per cápita es un factor clave para explicar la creación de empresas (Wennekers et al., 2005; Carree et al., 2007, Acs et al., 2007 y 2008), se utiliza como variable de control el Producto Interno Bruto (PIB) per-cápita, ajustado por la paridad de poder adquisitivo que se obtiene de la base de datos del Fondo Monetario Internacional (IMF), y la tasa de desempleo que se obtiene de la Organización Internacional del Trabajo (ILO). Asimismo, con la finalidad de controlar otras características asociadas al nivel de desarrollo económico, se incluyen variables binarias para agrupar los países según el nivel de ingresos: a) Ingreso alto OCDE, b) ingreso alto NO OCDE, c) ingreso medio-alto, d) ingreso medio bajo y bajo. En el Cuadro 3 se presenta una descripción detallada de las variables usadas en el análisis empírico.

Como se señaló previamente, en este trabajo la creación de empresas está condicionada por los factores del entorno, medidos a través de las instituciones informales y formales. Por lo tanto se plantea el siguiente modelo:

$T E A_{t t}=\alpha+\beta_{1} I I_{i t-1}+\beta_{2} I F_{i t-1}+\beta_{3} V C_{i t-1}+\varepsilon_{i t-1}$

donde:

IIt-1 representa la matriz de instituciones informales en el país i en el año $t$
$I F_{i t-1}$ se refiere a la matriz de instituciones formales en el país i en el año $t$ $V C_{i t-1}$ indica la matriz de variables de control en el país i en el año $t$

$\varepsilon_{i t-1}$ es la perturbación aleatoria que cumple los supuestos de partida.

Inicialmente se parte del enfoque más simple para analizar datos tipo panel, una regresión agrupada (pooled OLS) que consiste en omitir las dimensiones del espacio y el tiempo de los datos agrupados, calculando la regresión por mínimos cuadrados ordinarios MCO. Posteriormente, se estiman y comparan modelos de efectos aleatorios y efectos fijos. Los resultados del test de Hausman indican que el modelo de efectos aleatorios se ajusta mejor a los datos $\chi^{2}(8)=$ 4.89, Prob $\left.>\chi^{2}=0.7689\right)$, es decir que a pesar de que hay características particulares en cada país, éstas no son observables directamente, y en consecuencia, no pueden modelizarse como efectos fijos pero sí como aleatorios. El modelo también fue estimado introduciendo efectos temporales que capturen eventos comunes a todos los países, por ejemplo la crisis económica mundial. Los resultados de la prueba $F$ indican que las variables binarias temporales son significativas. Además, teniendo en cuenta que es muy probable que el nivel de creación de empresas en $t$ esté asociado con el nivel de creación de empresas en $t-1$, se aplicó el test de Wooldridge de autocorrelación de primer orden en los residuos. Los resultados indican que existen problemas de autocorrelación $(F(1,38)=4.820$, Prob $>F=$ .0343). Finalmente, dado que se detecta heteroscedasticidad, se estima un modelo para datos de panel con errores están- 


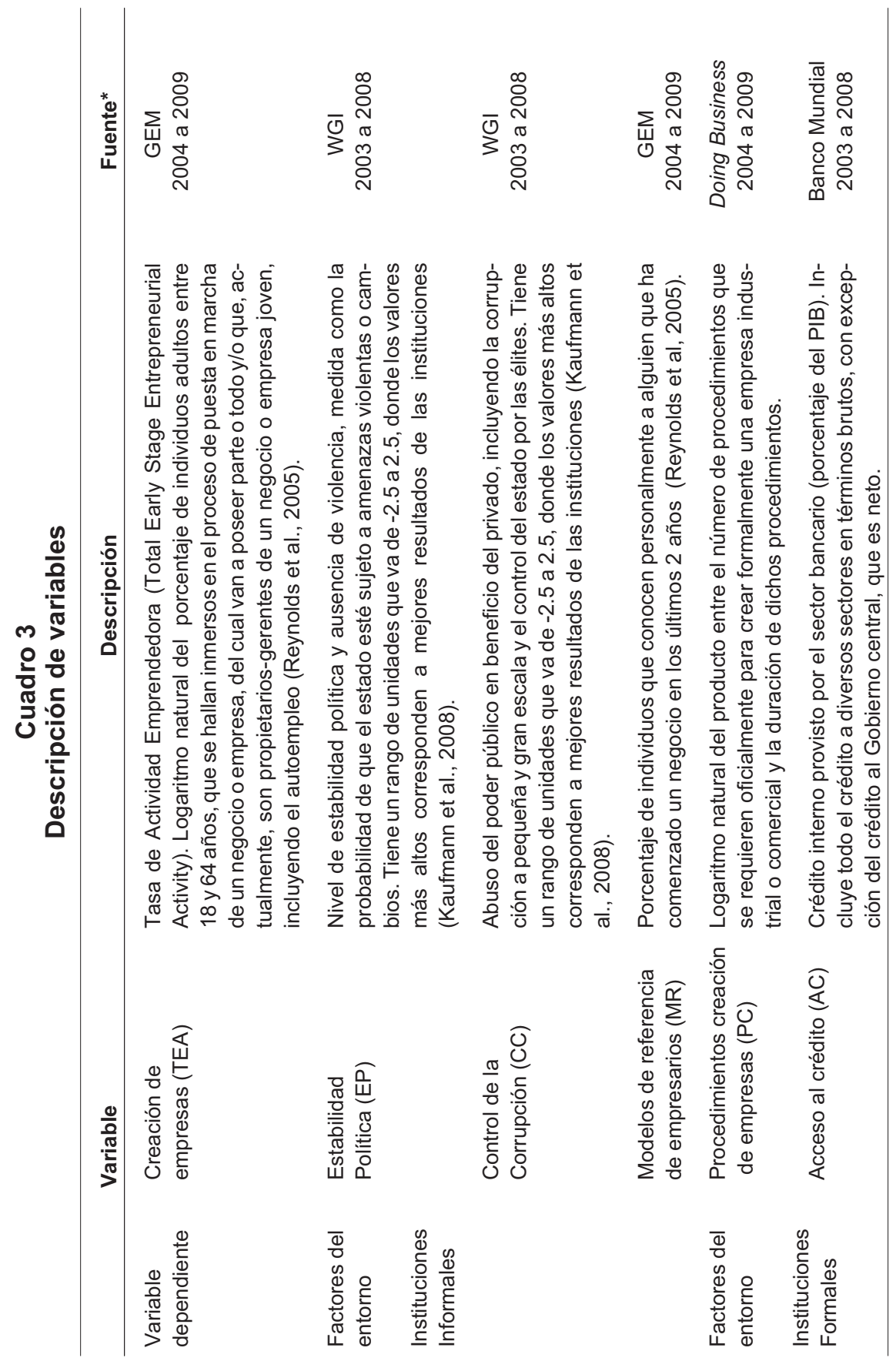




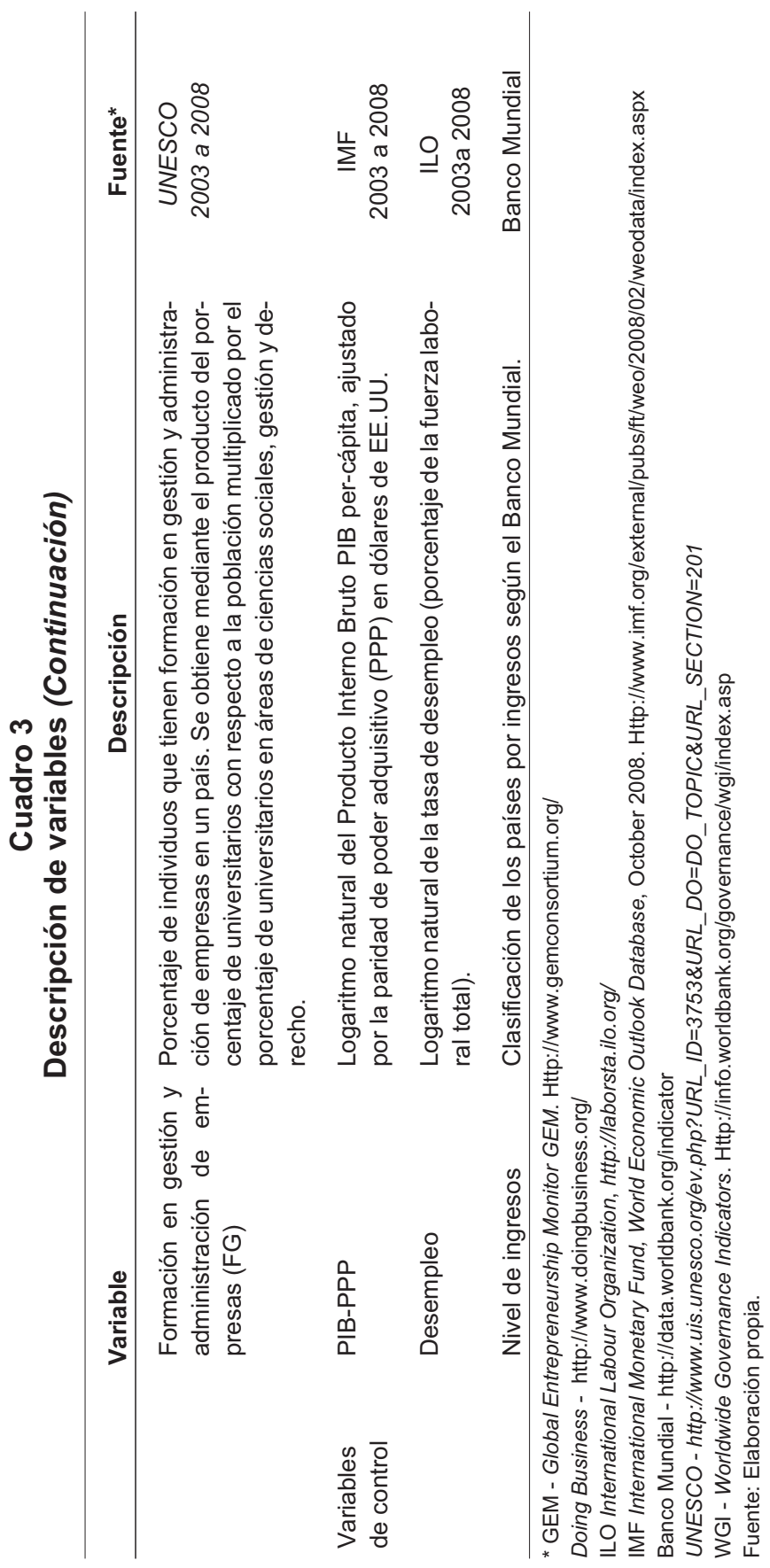




\section{Tabla 1}

\section{Estadísticos descriptivos y matriz de correlaciones}

\begin{tabular}{|c|c|c|c|c|c|}
\hline & Media & $\begin{array}{c}\text { Desv. } \\
\text { Estándar }\end{array}$ & 1. TEA & 2. EP & 3. $\mathrm{CC}$ \\
\hline 1. TEA & 9.77 & 7.06 & & & \\
\hline 2. Estabilidad Política (EP) & 0.31 & 0.84 & $-0.500^{* * *}$ & & \\
\hline 3. Control de la Corrupción (CC) & 0.77 & 1.05 & $-0.442^{\star \star \star}$ & $0.811^{* \star *}$ & \\
\hline 4. Modelos de Referencia (MR) & 42.24 & 10.61 & $0.515^{\star \star *}$ & $-0.197^{\star *}$ & $-0.235^{* * *}$ \\
\hline 5. Procedimientos (PC) & 5.05 & 1.31 & $0.283^{* * *}$ & $-0.500^{\star \star *}$ & $-0.616^{* * *}$ \\
\hline 6. Acceso al Crédito (AC) & 103.46 & 64.49 & $-0.343^{* * *}$ & $0.494^{* * *}$ & $0.602^{* * *}$ \\
\hline 7. Formación en Gestión (FG) & 2.45 & 1.27 & $-0.160^{*}$ & $0.134^{*}$ & $0.152^{*}$ \\
\hline 8. PIB-PPP & 2.90 & 0.76 & $-0.600^{* * *}$ & $0.732^{* * *}$ & $0.800^{* * *}$ \\
\hline 9. Desempleo & 1.93 & 0.52 & 0.020 & $-0.339^{* * *}$ & $-0.363^{* * *}$ \\
\hline
\end{tabular}

\section{MR}

5. PC

6. AC

7. FG

8. PIB-PPP

5. Procedimientos (PC)

6. Acceso al Crédito (AC)

7. Formación en Gestión (FG)

8. PIB-PPP

9. Desempleo

${ }^{* * *} p<0.001 ;{ }^{* *} p<0.01 ;{ }^{*} p<0.10$.

Fuente: Elaboración propia.

dar corregidos, en el que el término de error sigue un proceso autorregresivo de orden $1(A R(1))$.

La Tabla 1 muestra algunos estadísticos descriptivos (media y desviación estándar) de las variables consideradas, así como la matriz de correlaciones bivariadas a través del coeficiente de Pearson. Los resultados indican que la tasa promedio de actividad emprendedora es aproximadamente del $9 \%$, el nivel de estabilidad política promedio es de 0.31 , el control de la corrupción promedio es 0.77 y un $42 \%$ de los individuos conoce personalmente a alguien que ha creado una empresa en los últimos dos años. Así mismo, los procedimientos para crear una empresa son en promedio 8.3, con una duración de 32 días, el crédito interno promedio es de $101.87 \%$ del PIB y en promedio un $2.4 \%$ de los individuos tienen formación en gestión y administración de empresas.

La matriz de la Tabla 1 muestra algunas correlaciones interesantes entre las variables independientes, por ejemplo, puede verse una fuerte relación negativa y significativa entre los procedimientos para crear una empresa y el control de la corrupción. Tal resultado se evi- 
dencia en el caso de países como Brasil y Venezuela, que presentan el número más alto de procedimientos para crear una empresa (18 y 16 respectivamente, con una duración de 152 y 141 días en el 2009) y a su vez, tienen índices negativos en el control de la corrupción $(-0.11$ y -1.23 , respectivamente). Asimismo, puede verse que algunos coeficientes de correlación entre las variables independientes son superiores a 0.5 indicando posibles problemas de multicolinealidad, sin embargo, la prueba de multicolinealidad variance inflation factor (VIF) muestra valores aceptables -en este caso menores a 4.7- para las variables independientes, descartando que la colinealidad sea un problema grave.

Con la intención de aislar y comparar el efecto de las instituciones formales y las informales sobre la creación de empresas, se plantean varios modelos: el modelo 1 que incluye las instituciones formales y las variables de control; el modelo 2 con las instituciones informales y las variables de control; el modelo 3 que considera tanto instituciones informales como formales y las variables de control; el modelo 4 que presenta las instituciones informales y formales y las variables de control considerando la muestra de países de alto ingreso; y por último, el modelo 5 presenta las instituciones informales $y$ formales y las variables de control considerando la muestra de países de mediano y bajo ingreso (ver Tabla 2).

Como se mencionó anteriormente, el primer modelo considerado analiza el efecto de las instituciones formales (número de procedimientos para crear una empresa, formación en gestión y administración y acceso al crédito bancario) sobre la tasa de actividad emprendedora, cuando se controla el PIB-PPP, la tasa de desempleo y el grupo de ingresos del país. Los resultados indican que el impacto de las instituciones formales sobre la actividad emprendedora no es significativo. Específicamente, el número de procedimientos que se necesita para abrir una empresa no tiene influencia sobre la actividad emprendedora. Este resultado coincide con la evidencia empírica presentada por Van Stel et al. (2007), quien a su vez afirma que los emprendedores logran superar o evitar las cargas burocráticas, como el número de procedimientos o el tiempo que se requiere para poner en marcha una empresa, incluso si éstos son relativamente pesados. Así mismo el acceso al crédito y la formación en gestión y administración no tienen un impacto significativo sobre la tasa de creación de empresas.

Además, como era de esperar según la literatura sobre el tema (van Stel et al., 2005; Carree et al., 2007), existe una relación negativa significativa $(p<0.05)$ entre el PIB y la actividad emprendedora, relación que se confirma con las variables binarias relacionadas con el ingreso, que indican que la actividad emprendedora es mayor en los países de ingresos medio y bajo.

El segundo modelo muestra la relación entre las instituciones informales (estabilidad política, control de la corrupción y modelos de referencia) y la tasa de actividad emprendedora. Los resultados indican que dichas instituciones tienen un impacto estadísticamente significativo sobre la actividad emprendedora $(p<0.001)$. Analizando cada una de las variables, puede verse que la estabilidad 


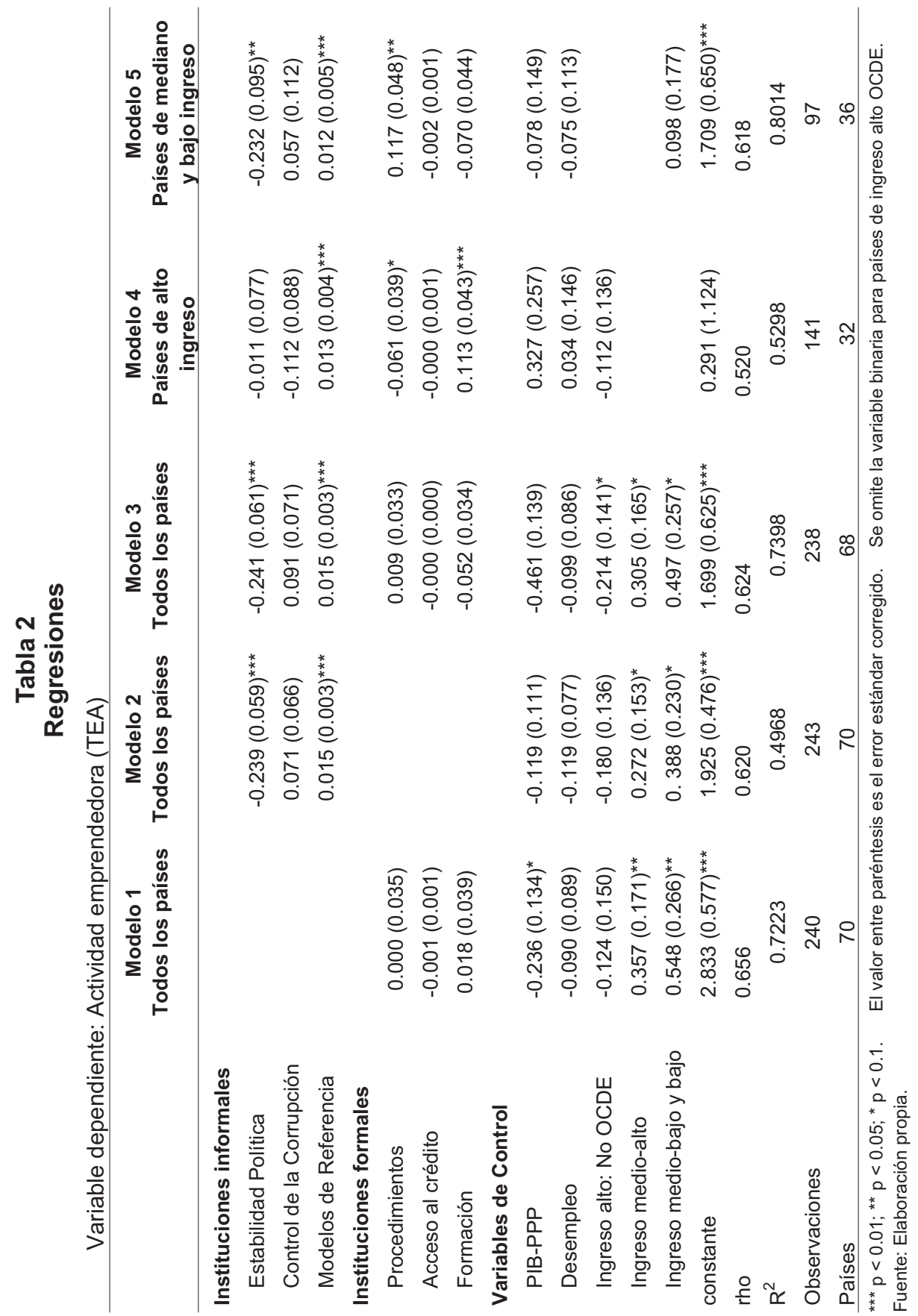


política tiene un impacto significativo en la actividad emprendedora, como se había propuesto en la hipótesis 1 , pero su signo es negativo; mientras que el control de la corrupción no influye significativamente sobre el emprendimiento. A su vez, la variable modelos de referencia tiene una relación positiva y significativa $(p<0.01)$ con la tasa de actividad emprendedora de los países considerados, resultados que están en la línea de la literatura que afirma que la presencia de emprendedores con experiencia y modelos de referencia exitosos transmiten mensajes positivos a los potenciales emprendedores (Gnyawali y Fogel, 1994) incrementando las tasas de creación de empresas. Las variables de control PIB-PPP y tasa de desempleo no son significativas, además, puede verse que, al igual que en el modelo 1 , los países de ingreso medio y bajo tienen tasas superiores de actividad emprendedora que los países de ingreso alto pertenecientes a la OCDE, usados como grupo de referencia.

En el tercer modelo se incluyen todas las variables consideradas en este trabajo, es decir, tanto las instituciones formales como los informales y las variables de control. Los resultados de la regresión muestran que las instituciones informales (estabilidad política y modelos de referencia) continúan siendo significativas y conservan los mismos signos que en el modelo 2, mientras que las instituciones formales no tienen una relación estadísticamente significativa con la actividad emprendedora. Se ratifica, entonces, la importancia de las instituciones informales para el fomento de la actividad emprendedora.
Con el fin de analizar mejor la relación entre instituciones informales y formales según el nivel de desarrollo económico de los países, en el cuarto modelo se presentan dichas instituciones y las variables de control, considerando únicamente la muestra de países de alto ingreso (tanto los pertenecientes a la OCDE como los que no). Puede verse que instituciones informales como estabilidad política y control de la corrupción no son significativas, mientras que los modelos de referencia tienen una relación positiva y significativa $(p<0.01)$ con la actividad emprendedora. Así mismo, instituciones formales como procedimientos y formación en gestión y administración tienen una relación significativa, la primera con signo negativo y la segunda con signo positivo. Lo anterior indica que en el caso de países de alto ingreso, mayores procedimientos para abrir una empresa desestimulan a los emprendedores, que probablemente optan por el trabajo por cuenta ajena. Así mismo, el signo positivo y significativo de la formación en gestión y administración indicaría que esta variable solo tiene impacto sobre la actividad emprendedora de los países de altos ingresos cuando interactúa con ciertas instituciones informales, por ejemplo, los modelos de referencia. Es decir, que altas tasas de universitarios con formación en gestión y administración implicarían que las personas cuentan con los conocimientos necesarios para crear una empresa; sin embargo, es necesaria la interacción con los modelos de referencia para influir positiva y significativamente sobre la actividad emprendedora. Se observa también que las variables de control PIB-PPP y tasa de desempleo no son 
significativas en esta muestra de países. El modelo considerado solamente explica el $53 \%$ de la variación de la actividad emprendedora de los países de ingreso alto.

Finalmente, en el quinto modelo se presentan las instituciones informales $y$ formales más las variables de control, considerando únicamente los países de ingreso medio y bajo. Puede verse que las instituciones informales, estabilidad política y modelos de referencia, son significativas y conservan el mismo signo que en los anteriores modelos, mientras que el control de la corrupción no es significativa. Así pues, menor estabilidad política y un porcentaje mayor de modelos de referencia incrementan la actividad emprendedora en los países de ingreso medio y bajo. Así mismo, las instituciones formales acceso al crédito y formación no tienen ninguna influencia sobre la actividad emprendedora, mientras que los procedimientos para crear empresas tienen una relación positiva y significativa con el emprendimiento. Este resultado, que contradice los supuestos considerados por el proyecto Doing Business, podría explicarse por las altas tasas de emprendimiento por necesidad que se presentan en estos países, donde un alto porcentaje de la población, de menores recursos económicos, se ve obligada a desarrollar una actividad empresarial para su subsistencia; incrementando el tamaño del sector informal de la economía que caracteriza a estos países. El $R^{2}$ indica que el quinto modelo explica el $80 \%$ de la variación total de la actividad emprendedora en los países de ingreso medio y bajo.

En términos generales, instituciones informales como la estabilidad políti- ca tiene una relación estadísticamente significativa con la actividad emprendedora, excepto en los países de alto ingreso; pero su signo es negativo, contrario a lo planteado en la hipótesis 1. Así mismo, el control de la corrupción no tiene influencia sobre la creación de empresas, por lo tanto se rechaza la hipótesis 2. Por último, acorde con la hipótesis 3 , la variable modelos de referencia tienen una relación positiva y significativa con la actividad emprendedora en todos los modelos considerados, tanto en la muestra de países de ingreso alto como en la muestra de ingresos medio y bajo.

Con respecto a instituciones formales como procedimientos para crear empresas, los resultados indican que en el caso de los países con altos ingresos la relación es negativa, mientras que en los países de ingreso medio y bajo dicha relación es positiva, así pues se rechaza la hipótesis 4 . Este resultado indicaría que las políticas y estrategias orientadas a la disminución de trámites para la creación de empresas no tienen un impacto sobre la tasa de actividad emprendedora sino sobre su distribución entre economía formal o informal, como bien lo apunta van Stel et al (2007). La variable acceso al crédito bancario no es significativa en ninguno de los modelos considerados, rechazando la hipótesis 5. Por último, también se rechaza la hipótesis 6 pues la formación en gestión y administración tiene un efecto positivo sobre la actividad emprendedora solamente en el caso de los países de alto ingreso, mientras que dicho efecto no es significativo en los países de ingreso medio y bajo. En el Cuadro 4 se presenta un resumen de los resultados del contraste de hipótesis. 


\section{Cuadro 4 \\ Resumen de los resultados empíricos}

H1: $\quad$ Existe una relación positiva entre la estabilidad política y la creación

Se rechaza de empresas.

H2: Existe una relación positiva entre el control de la corrupción y la crea-

Se rechaza ción de empresas.

H3: $\quad$ Existe una relación positiva entre los modelos de referencia de em- No se rechaza presarios y la creación de empresas.

H4: Existe una relación negativa entre el número de procedimientos para Se rechaza crear una empresa y la creación de empresas.

H5: Existe una relación positiva entre el acceso al crédito bancario y la Se rechaza creación de empresas.

H6: Existe una relación positiva entre el nivel de formación y gestión en No sechaza administración de empresas y la creación de empresas.

Fuente: Elaboración propia.

\section{Conclusiones}

Es bien reconocida la importancia de la creación de empresas por su aporte al desarrollo económico y social de los países y regiones. Así pues, mientras que las administraciones públicas muestran un particular interés en el diseño de políticas y estrategias para fomentar la actividad emprendedora, el sector académico se ha centrado en la comprensión de dicho fenómeno, analizando entre otras temáticas, las condiciones del entorno que afectan la creación de empresas.

En este contexto, el presente trabajo parte del enfoque institucional (North, 1990 y 2005) aplicado al ámbito de la creación de empresas a través del modelo de Gnyawali y Fogel (1994), para analizar la influencia que tienen las instituciones informales (estabilidad política, control de la corrupción y modelos de referencia) y las instituciones formales (procedimientos en creación de empresas, formación en gestión y administración, acceso al crédito bancario) en la actividad emprendedora, mediante un modelo de errores estándar corregidos para panel (Panel Corrected Standard Errors) para el periodo 2004-2009.

Los principales resultados del estudio indican que una menor estabilidad política y más modelos de referencia incrementan las tasas de actividad emprendedora. En términos generales, se observa que las instituciones formales tienen mayor impacto en los países de alto ingreso, mientras que las instituciones informales son más importantes en los países de ingreso medio y bajo. Este resultado puede entenderse a partir de la brecha existente entre las instituciones informales y formales, que es menor en los países de alto ingreso, caracterizados por valores compartidos acerca de la credibilidad de las normativas y la aceptación y cumplimiento de las leyes. En consecuencia, en entornos de este tipo, parece tener mayor sentido el fomento a la actividad emprendedora a partir de las instituciones formales, que serán 
acatadas por los miembros de la sociedad, y en particular por los emprendedores. Así mismo, en los países de ingresos medio y bajo, donde se siguen en menor medida las reglamentaciones establecidas y la sociedad sanciona menos el incumplimiento de las leyes, será a través de las instituciones informales (especialmente a través de una mayor difusión de los modelos de referencia), el mecanismo más adecuado para incentivar el emprendimiento.

En cuanto a futuras líneas de investigación, se propone el estudio de las diferencias entre los países de ingresos altos y los países de ingresos medio y bajo, tanto en lo referente a las características de la actividad emprendedora, como a los factores del entorno que la determinan. Dicho análisis permitiría avanzar en la definición de políticas y estrategias de fomento del emprendimiento, acorde con las condiciones específicas de los distintos países. En la misma línea, se podría avanzar en la caracterización de la actividad emprendedora y los factores que la condicionan, según el nivel de ingreso de los países, teniendo en cuenta la motivación y distinguiendo por oportunidad (creación de una empresa a partir de la percepción de que se puede explotar una oportunidad de negocio), versus por necesidad (iniciativa empresarial originada por la falta de otras posibles alternativas laborales, como por ejemplo, desempleo o insatisfacción con el trabajo anterior). Finalmente, se plantea seguir trabajando en el refinamiento y mejora de las variables utilizadas como aproximaciones de las instituciones. En este sentido, se reafirma la idoneidad de la teoría económica institucional como marco teórico más apropiado y robusto para el estudio de los factores del entorno que condicionan la creación de empresas.

\section{Referencias bibliográficas}

Acs, Zoltan y Szerb, Laszlo (2007). Entrepreneurship, economic growth and public policy. Small Business Economics, Vol. 28, No. 2, pp. 109-122.

Acs, Zoltan, Desai, Sameeksha y Hessels, Jolanda (2008). Entrepreneurship, economic development and institutions. Small Business Economics, Vol. 31, No 3, pp. 219-234.

Aidis, Ruta; Estrin, Saul y Mickiewicz, Tomasz. (2008). Institutions and entrepreneurship development in Russia: A comparative perspective. Journal of Business Venturing, Vol. 23, No. 6, pp. 656-672.

Aldrich, Howard E. y Zimmer, Catherine (1986). Entrepreneurship through Social Networks. En Donald L. Sexton y Ray W. Smilor (Eds.), The Art and Science of Entrepreneurship, pp. 3-23. New York: Ballinger.

Alvarez, Claudia y Urbano, David (2011a). Una década de investigación sobre el GEM: logros y retos. Academia, Revista Latinoamericana de Administración, No 46. pp. 1-22.

Alvarez, Claudia y Urbano, David (2011b). Environmental factors and entrepreneurial activity in Latin America. Academia, Revista Latinoamericana de Administración, No 48. pp. 126-139.

Alvarez, Claudia; Urbano, David; Coduras, Alicia y Ruiz, José (2011). Environmental conditions and entrepreneurial activity: A regional comparison in Spain. Journal of Small Business and Enterprise Development. Vol. 18, No 1. pp.120-140. 
Ang, James S. (1991). Small Business uniqueness and the theory of financial management. The Journal of Small Business Finance, Vol. 1, No. 1, pp. 1-13.

Anokhin, Sergey y Schulze, William S. (2009). Entrepreneurship, innovation, and corruption. Journal of Business Venturing, Vol. 24, No 5, pp. 465476.

Arenius, Pia y De Clercq, Dirk (2005). A network-based approach on opportunity recognition. Small Business Economics, Vol. 24, No 3, pp. 249265.

Arenius, Pia y Kovalainen, Anne (2006). Similarities and differences across the factors associated with women's selfemployment preference in the Nordic countries. International Small Business Journal, Vol. 24, No. 1, pp. 3159 .

Arenius, Pia y Minniti, Maria (2005). Perceptual variables and nascent entrepreneurship. Small Business Economics, Vol. 24, No. 3, pp. 233-247.

Audretsch, David B. (2004). Sustaining innovation and growth: public policy support for entrepreneurship. Industry and Innovation, Vol. 11, No. 3, pp. 167191.

Audretsch, David B. y Keilbach, Max (2004). Does entrepreneurship capital matter? Entrepreneurship: Theory \& Practice, Vol. 28, No 5, pp. 419-429.

Audretsch, David B. y Thurik, Roy (2001). What's new about the new economy? Sources of growth in the managed and entrepreneurial economies. Industrial and Corporate Change, Vol. 10, No 1, pp. 267-315.

Aysan, Ahmed F.; Nabli, Mustapha K. y Véganzonès-Varoudakis, MarieAnge. (2007). Governance institutions and private investment: an application to the Middle East and North Africa.
The Developing Economies, $\mathrm{Vol} 45$, No. 3, pp 339-377.

Bandura, Albert (1978). Reflections on selfefficacy. Advances in behavioral research and therapy, Vol. 1, No. 4, pp. 237-269.

Bates, Timothy (1997). Financing small business creation: the case of Chinese and Korean immigrant entrepreneurs. Journal of Business Venturing, Vol. 12, pp. 109-124.

Baughn, C. Christopher; Chua, Bee-Leng y Neupert, Kent E. (2006). The normative context for women's participation in entrepreneurship: a multicountry study. Entrepreneurship: Theory \& Practice, Vol. 30, No. 5, pp. 687-708.

Begley, Thomas M. y Tan, Wee-Liang (2001). The social-cultural environment for entrepreneurship: a comparison between East Asian and Anglo-Saxon Countries. Journal of International Business Studies, Vol. 32, No. 3, pp. 537-553.

Begley, Thomas M.; Tan Wee-Liang y Schoch, Herbert (2005). Politico-economic factors associated with interest in starting a business: a multi-country study. Entrepreneurship: Theory \& Practice, Vol. 29, No. 1, pp. 35-55.

Bennet, Robert y Robson, Paul (1999). The use of external business advise by SMEs in Britain. Entrepreneurship \& Regional Development, Vol. 11, pp. 155-180.

Berger, Brigitte (1991). The Culture of Entrepreneurship. San Francisco: ICS Press.

Bhave, Mahesh P. (1994). A process model of entrepreneurial venture creation. Journal of Business Venturing, Vol. 9, pp. 223-242.

Blanchflower, David G. y Oswald, Andrew J. (1998). What makes an entrepreneur? Journal of Labor Economics, Vol. 16, No. 1, pp. 26-60. 
Boyd, Nancy G y Vozikis, George S. (1994). The influence of self-efficacy on the development of entrepreneurial intentions and actions. Entrepreneurship: Theory \& Practice, Vol. 18, No. 4, pp. 63-77.

Brockhaus, Robert H. (1987). Entrepreneurial folklore. Journal of Small Business Management. Vol. 25, No 3, pp. 1-6.

Brockhaus, Robert H. y Horwitz, Pamela S. (1986). The psychology of the entrepreneur. En Donald L. Sexton y Raymond W. Smilor (Eds.), The art and science of entrepreneurship, pp. 25-48.Cambridge, MA: Ballinger.

Bruno, Albert V. y Tyebjee, Tyzoon T. (1982). The environment for entrepreneurship. En Calvin A. Kent, Donald L. Sexton y Karl H. Vesper. Encyclopedia of entrepreneurship, pp. 72-90.

Brush, Candida G. (1992). Research on women business owners: past trends, a new perspective and future directions. Entrepreneurship Theory \& Practice, Vol. 16, No. 4, pp. 5-30.

Bruton, Garry D.; Ahlstrom, David y Li, Han-Lin. (2010). Institutional Theory and Entrepreneurship: Where Are We Now and Where Do We Need to Move in the Future? Entrepreneurship: Theory \& Practice, Vol. 34, No. 3, pp. 421-440.

Busenitz, Lowell W.; West, G. Page; Shepherd, Dean; Nelson, Teresa; Chandler, Gaylen N. y Zacharakis, Andrew (2003). Entrepreneurship research in emergence: Past trends and future directions. Journal of Management, Vol. 29, No. 3, pp. 285-308.

Busenitz, Lowell W.; Gomez, Carolina y Spencer, Jennifer W. (2000). Country institutional profiles: unlocking entrepreneurial phenomena. Academy of Management Journal, Vol. 43, No. 5, pp. 994-1003.

Bygrave, Willian D. y Hofer, Charles W. (1991). Theorizing about Entrepreneurship.
Entrepreneurship: Theory \& Practice, Vol. 16, No. 2, pp. 13-22.

Carmignani, Fabrizio (2005). Efficiency of institutions, political stability and income. DynamicsEconWPA.

Carree, Martin; van Stel, André; Thurik, Roy y Wennekers, Sander (2007). The relationship between economic development and business ownership revisited. Entrepreneurship \& Regional Development, Vol. 19, No. 3, pp. 281291.

Carsrud, Alan L. y Johnson, Robyn W. (1989). Entrepreneurship: a social psychological perspective. Entrepreneurship \& Regional Development, Vol. 1, pp. 21-31.

Chen, Chao C; Greene, Patricia G. y Crick, Ann (1998). Does entrepreneurial selfefficacy distinguish entrepreneurs from managers? Journal of Business Venturing, Vol. 13, No. 4, pp. 295-316.

Collins, Orvis F.; Moore, David G. y Unwalla, Darab B. (1964). The Enterprising Man, MSU business studies.

Cooper, Arnold C. (1986). Entrepreneurship and high technology. En Donald L. Sexton y Raymond W. Smilor (Eds.). The art and science of entrepreneurship, pp. 153-180. Cambridge, MA: Ballinger.

Dana, Leo P. (1990). Saint Martin/Sint Maarten: a case study of the effects of culture on economic development. Journal of Small Business Management, Vol. 28, No. 4, pp. 91-98.

Dana, Leo P. (1996). Albania in the twilight zone: the perseritje model and its impact on small business. Journal of Small Business Management, Vol. 34, No. 1, pp. 64-70.

Davidsson, Per (1991). Continued entrepreneurship: ability, need and opportunity as determinants of small firm growth. Journal of Business Venturing, Vol. 6, No. 6, pp. 405-429. 
Davidsson, Per (1995). Culture, structure and regional levels of entrepreneurship. Entrepreneurship \& Regional Development, Vol. 7, pp. 41-62.

Davidsson, Per y Honig, Benson (2003). The role of social and human capital among nascent entrepreneurs. Journal of Business Venturing, Vol. 18, No. 3, pp. 301-331.

De Clercq, Dirk y Arenius, Pia (2006). The role of knowledge in business start-up activity. International Small Business Journal, Vol. 24, No. 4, pp. 339-358.

Delmar, Frédéric. y Davidsson Per (2000). Where do they come from? Prevalence and characteristics of nascent entrepreneurs. Entrepreneurship \& Regional Development, Vol. 12, No. 1, pp. 1-23.

Djankov, Simeon; Miguel, Edward; Qian, Yingyi; Roland, Gérard y Zhuravskaya, Ekaterina (2005). Who are the Russian entrepreneurs? Journal of European Economic Association Papers and Proceedings, Vol. 3, No. 23, pp. 587-597.

Djankov, Simeon; Qian, Yingyi; Roland, Gérard y Zhuravskaya, Ekaterina (2006). Entrepreneurship and development: first results for China and Russia. Paper presented at the American Economic Association Conference, Boston, MA.

Dolinsky, Arthur L.; Caputo, Richard K.; Pasumarty, Kishore y Quazi, Hesan (1993). The effects of education on business ownership: A longitudinal study of women. Entrepreneurship Theory and Practice, Vol. 18, No. 1, pp. 43-53.

Doucouliagos, Hristos y Ulubasoglu, Mehmet (2008). Democracy and economic growth: a meta-analysis. American Journal of Political Science, Vol. 52, No. 1, pp. 61-83.

Gartner, William B. (1985). A conceptual framework for describing the phenomenon of new venture creation.
Academy of Management Review, Vol. 10, No. 4, pp. 696-706.

Gartner, William B. y Vesper, Karl H. (1994). Experiments in entrepreneurship education: Successes and failures. Journal of Business Venturing, Vol. 9 , No. 3, pp. 179-187.

George, Gerard y Prabhu, Ganesh N. (2003). Developmental financial institutions as technology policy instruments: implications for innovation and entrepreneurship in emerging economies. Research Policy, Vol. 32, pp. 89-108.

Gnyawali, Devi R. y Fogel, Daniel S. (1994). Environments for entrepreneurship development: Key dimensions and research implications. Entrepreneurship: Theory \& Practice, Vol. 18, No 4, pp 43-62.

Gómez Haro, Samuel y Salmerón Gómez, Roman (2011). Influencia del entorno institucional en el desarrollo del emprendimiento español. Un análisis empírico. Revista Venezolana de Gerencia, Vol. 16, No 54, pp. 191-208.

Gorman, Gary, Hanlon, Dennis y King, Wayne (1997). Some research perspectives on entrepreneurship education. International Small Business Journal, Vol. 15, No 3, pp. 56-77.

Ho, Yuen-Ping y Wong, Poh-Kam (2007). Financing, regulatory costs and entrepreneurial propensity. Small Business Economics, Vol. 28, No. 2-3, pp. 187-204.

Holtz-Eakin, Douglas (1994). Sticking it out: Entrepreneurial survival and liquidity constraints. Journal of Political Economy, Vol. 102, No. 1, pp. 53-75.

Johannisson, Bengt (1988). Business formation: A network approach. Scandinavian Journal of Management, Vol. 4, pp. 83-99.

Johnson, Simon; Kaufmann, Daniel; McMillan, John y Woodruff, Christopher (2002). Property rights and finance. Ameri- 
can Economic Review, Vol. 92, pp, 1335-1356.

Johnson, Simon; McMillan, John y Woodruff, Christopher (1999). Contract enforcement in transition. EBRD Working paper No. 45. http://www.ebrd.org.

Johnson, Simon; McMillan, John y Woodruff, Christopher (2000). Entrepreneurs and the ordering of institutional reform. Economics of Transition, Vol. 8, No 1, pp. 1-36.

Kaufmann, Daniel y Kraay, Aart (2003). Governance and growth: Causality which way? - Evidence for the World. World Bank Institute, Washington, DC.

Kaufmann, Daniel, Kraay; Aart y Mastruzzi, Massimo (2008). Governance matters VII: Aggregate and individual governance indicators 1996-2007. World Bank Policy Research Working Paper. WPS 4654.

Koellinger, Philipp (2008). Why are some entrepreneurs more innovative than others? Small Business Economics, Vol. 31, No 1, pp. 21-37.

Koellinger, Philipp y Minniti, Maria (2006). Not for lack of trying: American entrepreneurship in black and white. Small Business Economics, Vol. 27, No 1, pp. 59-79.

Kortum, Samuel y Lerner, Josh (2000). Assessing the contribution of venture capital to innovation. The Rand Journal of Economics, Vol. 31, No 4, pp. 674-692.

Krueger, Norris (1993). The impact of prior entrepreneurial exposure on perceptions of new venture feasibility and Desirability. Entrepreneurship: Theory \& Practice, Vol. 18, No 1, pp. 5-21.

Krueger, Norris F.; Reilly, Michael D. y Carsrud, Alan L. (2000). Competing models of entrepreneurial intentions. Journal of Business Venturing, Vol. 15, pp. 411-432.
Kwon, Seok-Woo y Arenius, Pia (2010). Nations of entrepreneurs: A social capital perspective. Journal of Business Venturing, Vol. 25, No 3, pp. 315-330.

Langowitz, Nan y Minniti, Maria (2007). The entrepreneurial propensity of women. Entrepreneurship: Theory \& Practice, Vol. 31, No 3, pp. 341-364.

Levie, Jonathan (2007). Immigration, inmigration, ethnicity and entrepreneurship in the United Kingdom. Small Business Economics, Vol. 28, No. 2-3, pp. 143-169.

Levie, Jonathan y Autio, Erkko (2008). A theoretical grounding and test of the GEM model. Small Business Economics, Vol. 31, No 3, pp. 235-263.

Li, Hongyi; Xu, Lixin C. y Zou, Heng-fu (2000). Corruption, income distribution, and growth. Economics \& Politics, Vol. 12, No 2, pp. 155-181.

Lundstrom, Anders y Stevenson Lois A. (2005). Entrepreneurship policy: Theory and practice. International Studies in Entrepreneurship, Kluwer Academic Publishers.

Manolova, Tatiana S.; Eunni, Rangamohan V. y Gyoshev, Bojidar S. (2008). Institutional environments for entrepreneurship: Evidence from emerging economies in Eastern Europe. Entrepreneurship: Theory \& Practice, Vol. 32, No 1, pp. 203-218.

Marshall, J. Neill; Alderman, Neil; Wong, Cecilia y Thwaites, Alfred (1993). The impact of government- assisted management training and development on small and medium-sized enterprises in Britain. Environment and Planning C: Government and Policy, Vol. 11, No. 3, pp. 331-348.

Mason, Colin M. y Harrison, Richard T. (1994). The informal venture capital market in the UK. En Hughes, Alan y Storey, David J. (eds) Financing Small Firms. London: Routledge, pp. 64-111. 
Matthews, Charles H. y Moser, Steven B. (1995). Family background and gender: Implications for interest in small firm ownership. Entrepreneurship \& Regional Development, Vol. 7, No 4, pp. 365-377.

Maula, Markku; Autio, Erkko y Arenius, Pia (2005). What Drives Micro-Angel Investments? Small Business Economics, Vol. 25, No. 5, pp. 459-475.

McClelland, David C. (1961). The Achieving Society, Princeton: Van Nostrand.

McMillan, John y Woodruff, Christopher (1999). Interfirm relationships and informal credit in Vietnam. Quarterly Journal of Economics, Vol. 114, No 4, pp. 1285-1320.

McMillan, John y Woodruff, Christopher (2002). The central role of entrepreneurs in transition economies. Journal of Economic Perspectives, Vol. 16, No 3, pp. 153-170.

McMullen, Jeffery S.; Bagby, D. Ray y Palich, Leslie E. (2008). Economic freedom and the motivation to engage in entrepreneurial action. Entrepreneurship: Theory \& Practice, Vol. 32, No. 5, pp. 875-895.

McQuaid, Ronald W. (2002). Entrepreneurship and ICT industries: Support from regional and local policies. Regional Studies, Vol. 36, No 8, pp. 909-919.

Minniti, Maria y Nardone, Carlo (2007). Being in someone else's shoes: The role of gender in nascent entrepreneurship. Small Business Economics, Vol. 28, No. 2-3, pp. 223-238.

Naude, Wim; Gries, Thomas; Wood, Eric y Meintjies, Aloe (2008). Regional determinants of entrepreneurial startups in a developing country. Entrepreneurship \& Regional Development, Vol. 20, No 2, pp. 111-124.

North, Douglas C. (1990). Institutions, institutional change and economic per- formance. Cambridge: Cambridge University Press.

North, Douglas C. (2005). Understanding the process of economic change. Princeton: Princeton University Press.

Olsson, Ola (2000). Knowledge as a set in idea space: An epistemological view on growth. Journal of Economic Growth, Vol. 5, No. 3, pp. 253-276.

Parker, Simon C. (2004). The economics of self-employment and entrepreneurship, United Kingdom: Cambridge University Press.

Reynolds, Paul y White Sammis (1997). The entrepreneurial process: economic growth, women, and minorities. Quorum Books.

Reynolds, Paul; Bosma, Niels; Autio, Erkko; Hunt, Steve; De Bono, Natalie; Servais, Isabel; Lopez-García, Paloma y Chin, Nancy (2005). Global Entrepreneurship Monitor: Data collection design and implementation 1998-2003. Small Business Economics, Vol. 24, No 3, pp. 205-231.

Ritsilä, Jari y Tervo, Hannu (2002). Effects of unemployment on new firm formation: micro-level panel data evidence from Finland. Small Business Economics, Vol. 19, pp. 31-40.

Ronstadt, Robert (1985). The educated entrepreneurs: A new era of entrepreneurial education is beginning. American Journal of Small Business, Spring, pp. 37-53.

Roper, Stephen y Scott, Jonathan M. (2009). Perceived financial barriers and the start-up decision: An econometric analysis of gender differences using GEM data. International Small Business Journal, Vol. 27, No 2, pp. 149171.

Rose-Ackerman, Susan (2001). Trust, honesty, and corruption: Reflection of the state-building process. European 
Journal of Sociology, Vol. 42, No. 3, pp. 526-570.

Rose-Ackerman, Susan (2004). The challenge of poor governance and corruption. Copenhagen consensus challenge paper. Copenhagenconcensus.com.

Sarder, Jahangir H.; Ghosh, Dipak y Rosa, Peter (1997). The importance of support services to small enterprise in Bangladesh. Journal of Small Business Management, Vol. 35, No. 2, pp. 2636.

Scott, Michael G. y Twomey, Daniel W. (1988). The long-term supply of entrepreneurs: students' career aspirations in relation to entrepreneurship. Journal of Small Business Management, Vol. 26, No 4, pp. 5-13.

Shane, Scott y Kolvereid, Lars (1995). National environment, strategy, and new venture performance: A three country study. Journal of Small Business Management, April, pp. 37-50.

Shane, Scott y Venkataraman, Sankaran (2000). The promise of entrepreneurship as a field of research. Academy of Management Review, Vol. 25, No 1, pp. 217-226.

Shane, Scott; Kolvereid, Lars y Westhead Paul (1991). An exploratory examination of the reasons leading to new firm formation across country and gender. Journal of Business Venturing, Vol. 6, No 6, pp. 431-446.

Shapero, Albert y Sokol, Lisa (1982). The Social Dimensions of Entrepreneurship. En Kent, Calvin A., Sexton, Donald L. and Vesper, Karl H. (Eds.), Encyclopedia of Entrepreneurship, pp. 7290. Englewood Cliffs, N.J: PrenticeHall. Inc.

Spilling, Olav (1996). Regional variation of new firm formation: the Norwegian case. Entrepreneurship and Regional Development, Vol. 8, pp. 217-243.
Stephen, Frank; Urbano, David y van Hemmen, Stefan (2005). The impact of institutions on entrepreneurial activity. Managerial \& Decision Economics, Vol. 26, No 7, pp. 413-419.

Stephen, Frank, Urbano, David y van Hemmen, Stefan (2009). The responsiveness of entrepreneurs to working time regulations. Small Business Economics, Vol. 32, No. 3, pp. 259276.

Steyaert, Chris y Hjorth, Daniel (2006). Entrepreneurship as social change: A third new movements in Entrepreneurship, BookEdward Elgar.

Steyaert, Chris y Katz, Jerome (2004). Reclaiming the space of entrepreneurship in society: geographical, discursive and social dimensions. Entrepreneurship \& Regional Development, Vol. 16, No. 3, pp. 179-196.

Storey, David J. (1994). Employment. En Storey, David J. Understanding the small business sector, pp. 160-203, Ed. Routledge, London.

Szerb, Laszlo; Rappai, Gábor; Makra, Zsolt y Terjesen, Siri (2007). Informal investment in transition economies: Individual characteristics and clusters. Small Business Economics, Vol. 28, No. 2, pp. 257-271.

Thompson, Piers; Jones-Evans, Dylan y Kwong, Caleb (2009). Women and home-based entrepreneurship: Evidence from the United Kingdom. International Small Business Journal, Vol. 27, No 2, pp. 227-239.

Thornton, Patricia; Ribeiro-Soriano, Domingo y Urbano, David (2011). Sociocultural and entrepreneurial activity: An overview. International Journal of Small Business, Vol. 29, No. 2, pp. 105-118.

Tominc, Polona y Rebernik, Miroslav (2007). Growth aspirations and cultural support for entrepreneurship: A compari- 
son of post-socialist countries. Small Business Economics, Vol. 28, No 2, pp. 239-255.

Trulsson, Per. (2002). Constraints of growthoriented enterprises in the southern and eastern African region. Journal of Developmental Entrepreneurship, Vol. 7, No 3, pp. 331-339.

Urbano David; Díaz, Carlos y Hernández, Ricardo (2007). Evolución y principios de la Teoría Económica Institucional. Una propuesta de aplicación para el análisis de los factores condicionantes de la Creación de Empresas. Investigaciones Europeas de Dirección y Economía de la Empresa, Vol. 13, No 2, pp. 183-198.

Urbano, David (2006). La creación de empresas en Catalunya. Organismos de apoyo y actitudes hacia la actividad emprendedora. Barcelona: Col.lecció d'estudis CIDEM.

Vaillant, Yancy y Lafuente, Esteban (2007). Do different institutional frameworks condition the influence of local fear of failure and entrepreneurial examples over entrepreneurial activity?. Entrepreneurship \& Regional Development, Vol. 19, No 4, pp. 313-337.

van Auken, Howard E. (1999). Obstacles to Business Launch. Journal of Developmental Entrepreneurship, Vol. 4, No 2, pp. 175-187.

van Gelderen, Marco; Thurik, Roy y Bosma, Niels. (2006). Success and risk factors in the pre-startup phase. Small Business Economics, Vol. 26, No. 4, pp. 319-335.

van Stel, André; Carree, Martin y Thurik, Roy (2005). The effect of entrepreneurial activity on national economic growth. Small Business Economics, Vol. 24, No. 3, pp. 311-321.

van Stel, André; Storey, David J. y Thurik, Roy (2007). The effect of business regulations on nascent and young business entrepreneurship. Small Business Economics, Vol. 28, No 2-3, pp. 171186.

Veciana, José María y Urbano, David (2008). The institutional approach to entrepreneurship research: Introduction. International Entrepreneurship and Management Journal, Vol. 4, No 4, pp. 365-379.

Verheul, Ingrid; van Stel, André y Thurik, Roy (2006). Explaining female and male entrepreneurship at the country level. Entrepreneurship \& Regional Development, Vol. 18, No 2, pp. 151-183.

Verheul, Ingrid; Wennekers, Sander; Audretsch, David B. y Thurik, Roy (2001). An eclectic theory of entrepreneurship: policies, institutions and culture. En Audretsch, David B. (Ed.), SMEs in the Age of Globalization, pp. 533-606. Cheltenham: Edward Elgar Publishing.

Wagner, Joachim (2007). What a difference a $Y$ makes-female and male nascent entrepreneurs in Germany. Small Business Economics, Vol. 28, No 1, pp. 1-21.

Welter, Friederike (2005). Entrepreneurial behavior in differing environments. En David B. Audretsch, Heike Grimm y Charles W. Wessner (Eds.), Local heroes in the global village globalization and the new entrepreneurship policies, pp. 93-112. International Studies in Entrepreneurship. New York: Springer.

Welter, Friederike (2011). Contextualizing Entrepreneurship - Conceptual Challenges and Ways Forward. Entrepreneurship: Theory \& Practice, Vol. 35, No 1, pp. 165-184.

Welter, Friederike y Smallbone, David (2011). Institutional perspectives on entrepreneurial behaviour in challenging environments. Journal of Small Business Management, Vol. 49, No. 1, pp. 107-125. 
Wennekers, Sander, y Thurik, Roy (1999). Linking entrepreneurship and economic growth. Small Business Economics, Vol. 13, No. 1, pp. 27-55.

Wennekers, Sander; van Stel, André; Thurik, Roy y Reynolds, Paul (2005). Nascent entrepreneurship and the level of economic development. Small Business Economics, Vol. 24, No. 3, pp. 293309.

Wetzel, William E. (1987). The informal venture capital market: aspects of scale and market efficiency. Journal of Business Venturing, Vol. 2, No. 4, pp. 299-313.

Wohlgemuth, Michael (2000). Political entrepreneurship and bidding for political monopoly. Journal of Evolutionary Economics, Vol. 10, pp. 273-295.

Young, Earl y Welsch Harold (1993). Major elements in entrepreneurial development in central Mexico. Journal of Small Business Management, Vol. 31, No. 4, pp. $80-85$. 